\title{
Pêches des bélugas, des anguilles et des esturgeons noirs de l'estuaire du St-Laurent
}

Entre la protection et la marchandisation

Beluga Whale, Eel, and Black Sturgeon Fishery in the St Lawrence Estuary

(Québec): An Activity Enmeshed between Protection and Commodification

\section{Sabrina Doyon}

\section{OpenEdition}

Journals

Édition électronique

URL : http://journals.openedition.org/ethnoecologie/3472

DOI : $10.4000 /$ ethnoecologie.3472

ISSN : 2267-2419

\section{Éditeur}

Laboratoire Eco-anthropologie et Ethnobiologie

\section{Référence électronique}

Sabrina Doyon, «Pêches des bélugas, des anguilles et des esturgeons noirs de l'estuaire du St-Laurent », Revue d'ethnoécologie [En ligne], 13 | 2018, mis en ligne le 25 juin 2018, consulté le 21 avril 2019. URL : http://journals.openedition.org/ethnoecologie/3472 ; DOI : 10.4000/ethnoecologie.3472

Ce document a été généré automatiquement le 21 avril 2019.

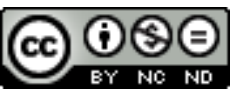

Revue d'ethnoécologie est mis à disposition selon les termes de la licence Creative Commons Attribution - Pas d'Utilisation Commerciale - Pas de Modification 4.0 International. 


\title{
Pêches des bélugas, des anguilles et des esturgeons noirs de l'estuaire du St-Laurent
}

\author{
Entre la protection et la marchandisation \\ Beluga Whale, Eel, and Black Sturgeon Fishery in the St Lawrence Estuary \\ (Québec): An Activity Enmeshed between Protection and Commodification
}

\section{Sabrina Doyon}

1 Cet article dresse un portrait de l'exploitation, de la conservation et de la mise en marché de trois espèces emblématiques de l'estuaire du St-Laurent, le béluga ${ }^{1}$, l'anguille et l'esturgeon noir, dont la pêche est désormais en péril. L'anguille et l'esturgeon noir sont les deux dernières espèces pêchées commercialement dans ce secteur de l'estuaire et les savoirs, les techniques et les équipements de ces deux pêches s'appuient sur un savoir qui a été développé grâce à la pêche au béluga, maintenant interdite. Les études anthropologiques concernant ces pratiques sont très limitées, et il s'avère intéressant de les documenter et d'interroger le contexte de leur déclin.

2 À l'intérêt ethnographique d'étudier ces espèces s'ajoute un intérêt disciplinaire. L'étude des pêches d'espèces spécifiques irrigue le champ de l'anthropologie maritime depuis longtemps. Des exemples notoires concernent la pêche à la morue et aux poissons de fonds dans l'Atlantique (Acheson 2011, Acheson \& Gardner 2004, Bavington 2010, Davis 2014, Davis et al. 2006) et la pêche au flétan, à l'églefin et au saumon dans le pacifique nord (Hébert 2015, Menzies 2012, Pinkerton 2013). Ces travaux ont permis d'examiner les dimensions sociales, économiques, politiques et symboliques propres aux différents aspects de ces pêches spécifiques, tels que les équipements, le temps de travail et la répartition des revenus, les écosystèmes, les organisations locales et les mécanismes de gestion.

3 Cet angle de recherche est remis à l'avant-scène dans le contexte des changements climatiques et du réchauffement des océans. Des études récentes documentent la disparition de certaines espèces et le déplacement d'autres de zones méridionales vers 
des zones plus nordiques, bouleversant tant les écosystèmes marins que les pratiques de pêche et les systèmes sociaux qui les portent (Pauly \& Zeller 2016, Watson \& Pauly 2013). Les changements affectant les espèces marines et leurs pêches, bien que moins spectaculaires que ceux décrits par les chercheurs de l'Institute for the Oceans and Fisheries, ont cependant eu lieu de tout temps. Comme cet article cherche à le présenter, ils témoignent de processus complexes où les transformations environnementales sont liées à des enjeux politiques qui peuvent être éclairés par l'approche de la political ecology.

4 La political ecology, qui allie des préoccupations pour l'écologie et l'économie politique (Blaikie \& Brookfield 1987), s'intéresse aux sources contextuelles des changements environnementaux, aux enjeux locaux pour l'accès à la nature et aux ramifications politiques des changements environnementaux (Bryant \& Bailey 1997) marquant la production des lieux et des paysages que supposent ces processus (Brockington \& Duffy 2010, Peet et al. 2011). En anthropologie maritime, elle a notamment été mise à profit afin d'éclairer les enchevêtrements des développements industriels des pêches, la privatisation par l'établissement de quotas de pêche (Carothers 2008, Carothers \& Chambers 2012, Mansfield 2004, 2010, McCay 1995, Olson 2011, Wiber 2000), les luttes locales pour l'accès aux zones de pêche (Andreatta \& Parlier 2010, Charest, Girard \& Rodon 2012, Doyon 2002, Fraga et al. 2008).

5 Cette approche sera mobilisée dans cet article afin, premièrement, d'éclairer les conséquences environnementales du développement capitaliste dans l'histoire récente des pêches de bélugas, d'anguilles et d'esturgeons. Deuxièmement, les politiques de gestion et de conservation, ancrées dans les transformations écologiques et les logiques marchandes, seront explorées. Enfin, l'examen les enjeux d'accès aux ressources, tant marines, financières, politiques, qu'humaines, rencontrés par les pêcheurs, et leurs conséquences pour la survie de ces pêches, sera proposé, mettant en lumière les liens étroits entre la protection de ces espèces et leur marchandisation.

6 Les pêches étudiées se pratiquent au Kamouraska, l'une des huit municipalités régionales de comté (MRC) de la région administrative du Bas-St-Laurent. Le Kamouraska, dont la superficie est de 2244 hectares, compte une population de 21154 habitants, soit près de $11 \%$ de la région du Bas-St-Laurent, dont la majorité habite la bande côtière (Gouvernement du Québec 2011). L'activité principale est l'agriculture, et plus particulièrement la production laitière. La coupe de bois de sciage et de bois de pâte est également une source de revenu non négligeable pour ceux qui habitent dans les hautes terres (Fortin \& Lechasseur 1993). La pêche demeure une activité importante dans la région du Bas-St-Laurent, bien que nettement moindre que dans les régions de la Gaspésie, des Îles-de-la-Madeleine ou de la Côte-Nord. La valeur des débarquements en 2008 s'élève à 9,4 millions de dollars, représentant environ $10 \%$ de la valeur des débarquements des pêches du Québec (Gouvernement du Canada 2015). Les pêches du Bas-St-Laurent sont surtout représentées par la pêche à la crevette nordique et au crabe des neiges, qui sont très lucratives et dont les débarquements se font dans les MRC de Rimouski et de Matane. Les débarquements et la valeur des pêches de la région de Kamouraska sont minimes en comparaison.

7 Il n'en a cependant pas toujours été ainsi et la pêche y a déjà été une activité florissante, constituant un pivot culturel et économique de la région. En effet, toutes les espèces de poissons pouvaient, jusque dans les années 1980, y être capturées et elles ont pendant des siècles offert un moyen de survivre aux hivers rigoureux puis de s'insérer dans l'économie de marché. Comment cette activité, caractérisée par une technique de pêche à 
gué, a-t-elle pu se transformer si radicalement? Cet article tentera de comprendre cette situation en présentant d'abord l'évolution de la pêche au béluga qui a permis l'avènement des pêches d'anguilles et d'esturgeons, puis le processus de dégradation environnementale qui a mené à des politiques de gestion ayant contribué à marginaliser les pêcheurs et, enfin, à la manière dont cette situation est maintenant liée à une patrimonialisation de ces espèces et à de nouveaux mécanismes de marchandisation.

\section{Béluga : de la pêche d'un prédateur carnassier à la conservation d'un mammifère bienveillant}

8 Le béluga est un animal emblématique du fleuve qui y a toujours été pêché, tant par les autochtones que par les colons français. Jacques Cartier rapporte en avoir vu dès son arrivée dans les eaux de l'estuaire. Les navigateurs qui terminaient leur traversée de l'Atlantique se délectaient de cette chair fraîche après des semaines de consommation de nourriture salée et séchée (Franck 2008).

9 Les premiers colons ont pêché le béluga sur les rives du St-Laurent, en bateau ou à gué. Dans la région du Bas-St-Laurent les nouveaux arrivés, dont les terres avaient été octroyées par la couronne et qui avaient le titre de seigneur, avaient tous les droits sur les espèces du fleuve faisant face à leurs terres. Cette appropriation fut l'objet de contestations et d'actes de résistance, alors que les habitants des terres seigneuriales ont revendiqué l'accès aux ressources du fleuve qui se trouvaient devant leur propriété et gagné le droit de les exploiter (Musée de la mémoire vivante 2009). Ce type d'appropriation demeure en vigueur encore aujourd'hui avec la pêche à l'anguille et à l'esturgeon.

Différentes techniques de pêche ont été mises en œuvre dans le fleuve St-Laurent pour capturer le béluga (Vladykov 1944). La pêche avec un filet maillant et la chasse avec une arme à feu ou un harpon à bord d'un bateau à moteur ou à voile était pratiquée sur la côte nord du fleuve (Pointe-Lebel, les Escoumins, lîle-aux-Coudres). À Rivière-Ouelle, sur la rive sud, les fascines étaient utilisées dès 1720 .

11 Pour ce faire, les pêcheurs disposaient dans l'estran 7000 troncs de petits arbres de quatre mètres de haut dans un demi-cercle de plus de 2000 mètres (38 arpents) espacés de 40 centimètres chacun. Les bélugas s'y engageaient et y restaient captifs lors des changements de marées, puis étaient harponnés. Les pêcheurs décrivaient les animaux pêchés comme étant gentils, naïfs, curieux et faciles à tuer parce que "sans malice " (Vladykov 1940).

12 L'activité de pêche aux bélugas s'est maintenue pendant deux siècles à un rythme plutôt lent, car l'abondance fluctuante du mammifère ne permettait pas une régularité des prises et ne favorisa pas l'industrialisation, comme ce fut le cas avec les autres activités halieutiques et d'exploitation des ressources primaires du Québec (Innis 1956). Cette pêche, qui offrait néanmoins des revenus intéressants, a permis en 1870 la création d'une société en commandites, une des premières tenue par des francophones au Québec. La société comptait 7200 parts valant chacune un dollar; chaque actionnaire devait entretenir une partie de la fascine correspondant au nombre d'actions possédées et recevait les redevances de la vente des captures (Musée de la mémoire vivante 2009).

Dès la fin $d u X^{e} x^{e}$ siècle, les bélugas pêchés par cette compagnie étaient recherchés principalement pour leur gras, une ressource motivant également les grandes pêches de 
cétacés dans les mers du monde à cette époque et auxquelles prenaient part les Britanniques, les principaux acheteurs des bélugas du Québec. L'Angleterre achetait ainsi la production d'huile de béluga du fleuve St-Laurent, qui était ensuite envoyée par bateau dans la métropole. Cette huile animale fut plus tard, dans la première moitié $\mathrm{du} \mathrm{xx}^{\mathrm{e}}$ siècle, envoyée par train aux États-Unis. La chasse aux bélugas répondait à une demande internationale et permettait le développement capitaliste des grandes métropoles et de leur empire colonial.

\section{De l'extermination systématique du « dévoreur des profondeurs » à sa conservation}

À la fin du XIX siècle, un changement perturbe le processus de développement de l'exploitation des ressources naturelles du Québec. Les pêcheurs du fleuve constatent une diminution des prises des espèces commerciales lucratives telles que la morue. Les représentants du clergé, alors très impliqués dans toutes les affaires de la vie quotidienne des habitants ainsi qu'en politique, et par extension dans la gestion des ressources naturelles, s'immiscent dans le débat. Ils militent en faveur de la destruction et de l'extermination des bélugas, considérés comme des dévoreurs de morues et de saumons, planche de salut des colons. Le béluga devient dès lors une espèce à abattre, littéralement (Franck 2008). Dans les années 1920, le clergé fit pression sur le gouvernement Taschereau et son ministre à vie des pêches (Musée de la mémoire vivante 2009). Ils font valoir l'importance de stimuler le développement des économies régionales afin de leur permettre d'atteindre leur plein potentiel. Les politiques de gestion mises en place doivent favoriser le développement des pêches les plus lucratives, dont les systèmes de transformation ont nécessité des investissements financiers d'entreprises nationales et internationales, comme c'est le cas avec la pêche à la morue.

La guerre au béluga est déclarée. Le ministère de la Colonisation, des Mines et des Pêcheries distribue des carabines et des munitions aux pêcheurs pour qu'ils abattent tous les marsouins qu'ils voient. Chaque marin qui apporte son arme, lorsqu'il prend la mer, reçoit une allocation mensuelle de 30 \$. Cette mesure incitative est rapidement bonifiée, entre 1928 à 1939, par une prime de $15 \$$ remise à quiconque rapporte une queue de marsouin à un représentant du ministère concerné. Ce Far Ouest maritime ne semble pas assez efficace aux dires des pêcheurs de morue. D'autres pressions suivent et le gouvernement prend les grands moyens. En cette période d'entre-deux guerres, de nouvelles technologies sont accessibles. C'est du haut des airs que les troupeaux de bélugas sont chassés. Un pilote est engagé pour les bombarder à partir d'un petit avion de chasse. (Franck 2008)

Les recensements de captures montrent rapidement que le nombre de béluga diminue. La société en commandite, qui a été rachetée par un résident de Rivière-Ouelle (Musée de la mémoire vivante 2009), a certes réalisé une pêche miraculeuse en 1929, lors de laquelle 112 bêtes ont été tuées pendant une seule pêche et 187 bêtes furent capturées en une saison, mais après cette date les captures se font rares et de plus en plus difficiles. Cette diminution ne permet pas au marché du béluga de se consolider.

17 Les débouchés pour les produits du béluga sont alors pourtant croissants. Le cuir de béluga est reconnu pour sa qualité et sa solidité. Les lacets faits dans sa peau sont particulièrement recherchés. La peau, extrêmement résistante permettait la confection de vêtements, de chaussures et de sacs. L'huile de béluga, qui contient des acides gras 
libres, est d'une qualité recherchée car elle ne s'altère pas. Cette graisse fondue servait surtout de combustible pour les lampes et connut une période florissante avec l'avènement du chemin de fer; l'huile de béluga servait alors pour la lubrification des roues de locomotives et de wagons. L'huile provenant de la tête du béluga, plus fine, servait quant à elle à la lubrification de mécanismes délicats, plus particulièrement l'horlogerie et l'armement. La viande était vendue aux éleveurs d'animaux à fourrure. Un béluga de taille moyenne pouvait valoir, en considérant le prix de l'huile, de la peau et de la viande, environ 30 \$ en 1943; le prix du litre d'huile de béluga a oscillé dans la première moitié du XIX ${ }^{e}$ siècle entre 13 sous le litre d'huile et 6,70 \$ pour celui « d'huile de tête ». Le gouvernement canadien cherchait de plus à ouvrir de nouveaux débouchés économiques et a envisagé de développer la pêche sportive de bélugas, dans le même esprit que la pêche au saumon, pour de riches Américains venant profiter des attraits naturels de la « belle province » (Vladykov 1944).

18 Le gouvernement du Québec, par le Département des pêcheries du Québec, entreprend dans les années 1930 des recherches approfondies sur ce mammifère et publia une série d'études sur la biologie des bélugas du fleuve et du golfe du St-Laurent, sur leur incidence sur la pêche commerciale de poissons ainsi que sur leur potentiel économique (Vladykov 1940, 1943). Le chercheur principal du projet embaucha des médecins et des représentants gouvernementaux qui s'établissaient dans les villages côtiers pour les périodes estivales et les mit en charge de recenser des informations concernant les bélugas. Les études révélèrent que l'alimentation du béluga ne consistait pas en des espèces commerciales et, après la guerre, la pêche aux bélugas est abandonnée.

Cette situation est également à mettre en lien avec l'avènement du Conseil mondial de protection des baleines. Cet organisme, fondé après la deuxième guerre, vise la protection de ces mammifères pour en assurer une exploitation pérenne. Il fut d'abord constitué uniquement de membres provenant des pays qui participaient à la chasse aux baleines pour ensuite inclure des pays non-producteurs. Après une évaluation des stocks mondiaux de cétacés, un moratoire fut mis en place et des quotas instaurés. Suite à ces mesures de gestion, certains pays, dont l'Angleterre, se retirèrent des activités de pêche et de mise en marché des produits de baleine, une activité qui n'est alors plus jugée rentable dans ce contexte et au vu de l'avènement des huiles de synthèse, de l'exploitation des hydrocarbures et de la transformation du pétrole.

20 Au Canada, tous les types de pêche au béluga, commerciale et sportive, sont prohibés en 1978 et les produits du béluga sont interdits de commercialisation et de consommation ${ }^{2}$. L'espèce fut désignée en voie de disparition en 1983 par le Comité sur la situation des espèces en péril au Canada (COSEPAC). Ce statut fut toutefois révisé en 2004 et le béluga est alors catégorisé simplement en tant qu'espèce menacée. Il devint par ailleurs l'icône du nouveau parc marin du Saguenay, d'une superficie de 1245 km carrés créé entre 1989 et 1998. Grâce à cette nouvelle aire protégée, les activités touristiques d'observation de bélugas sur le fleuve sont plus encadrées et sa précarité est reconnue par différents organismes, dont le Groupe de recherche et d'éducation sur les mammifères marins (GREMM) qui mit en place un programme d'adoption symbolique d'un béluga pour 5 $000 \$$. Il figure également sur les listes rouges des grands organismes internationaux de protection de la nature.

21 En 2013, le béluga a repris le devant de la scène de façon spectaculaire. Il est devenu l'acteur principal d'un débat autour de la construction d'un port en eau profonde dans le fleuve, où serait acheminé par pipeline le pétrole des sables bitumineux de l'Ouest du 
Canada pour y être transbordé par bateau. Or, la construction de ce port se trouve dans la zone de reproduction et d'élevage des bélugas, désignée comme leur "pouponnière », et menace leur survie. Au nom de la protection du béluga, de nombreux groupes environnementaux et de la société civile ont milité afin de faire valoir les droits de cette espèce et l'importance de la protection du fleuve contre des marées noires. Leurs démarches, conjuguées à un changement de statut par le COSEPAC, faisant du béluga à nouveau une espèce en voie de disparition, a fait en sorte que le projet de port en eau profonde a été suspendu puis interdit.

En passant d'un réservoir d'huile à extraire à l'emblème charismatique ultime du fleuve, le béluga démontre bien que les perceptions de ce qui est à protéger changent d'une époque à l'autre, et que cette perception est par ailleurs liée aux processus et possibilités économiques d'un moment historique. Aujourd'hui, le béluga, devenu une espèce en péril, est l'indicateur de la santé du St-Laurent, le témoin impuissant de l'obsession humaine pour le développement et la victime de la mondialisation et des ports en eaux profondes qu'elle suppose désormais. Le béluga est passé d'un statu d'un pseudo-poisson nuisible à celui de réserve de carburant puis d'alter-ego marin d'Homo sapiens.

La protection du béluga s'inscrit dans un contexte où la commercialisation de cette espèce était rendue difficile par les fluctuations de la pêche et par les transformations industrielles permettant le remplacement des huiles animales par des huiles de synthèse. La fin de l'âge d'or de la pêche au béluga correspond également au moment où s'intensifie la pêche à l'anguille, puis à l'esturgeon noir. L'organisation socio-économique qui a structuré la pêche au béluga a évolué autour d'un système de tenure foncière et de droits d'exploitation des fascines et de la création de la société en commandite, ce qui a facilité la transition vers une pêche commerciale plus soutenue de l'anguille et de l'esturgeon.

\section{Les anguilles et les esturgeons noirs de l'estuaire du St-Laurent : précarisation des ressources, gestion halieutique et marginalisation des pêches}

Les pêches d'anguilles et d'esturgeon noir ont été très généreuses historiquement. Pendant des siècles, ces espèces ont été pêchées dans le St-Laurent et son bassin versant. Les premiers colons français rapportent que les autochtones pêchaient déjà l'anguille et l'esturgeon à leur arrivée. Jusque dans les années 1950, les anguilles ont été la deuxième espèce la plus pêchée au Québec après la morue. La très probable disparition de ces pêches, conséquente à la possible extinction de ces espèces, a été annoncée depuis plusieurs décennies. Les populations d'anguilles sont maintenant à moins de $1 \%$ de leur population originelle (en 2010-2011, seulement 155000 femelles adultes auraient migré vers la mer des Sargasses) (Robitaille \&Tremblay 1994, Caron et al. 2007, Verreault et al. 2012) et l'esturgeon noir est désigné en tant qu'espèce menacée au Canada et en voie d'extinction au États-Unis; seule la population québécoise fait exception (Verreault \& Trencia 2011).

L'industrialisation du St-Laurent et des Grands Lacs est une source importante de contamination et une des principales causes de la réduction des populations d'anguilles et d'esturgeons. Les industries militaires, de pâtes et papiers, de l'automobile et de l'aluminium ont pollué le fleuve et ses ressources avec des contaminants, dont des bisphénols polychlorés et des organochlorés. L'épandage aérien de pesticides (DDT) pour 
éliminer les éclosions de moustiques à l'occasion de l'Exposition Universelle de Montréal en 1967, et possiblement le dragage et le renflouage lié à l'île Ste-Hélène et au métro de Montréal, ont eu des impacts très néfastes sur ces espèces, menant à la quasi-disparition de l'esturgeon noir jusque dans les années 1980. De plus, les obstructions des 8400 barrages présents dans le bassin versant du St-Laurent et les grandes turbines hydroélectriques au fil de l'eau de la rivière des Outaouais ont anéanti la population de son bassin versant ; celles de Moses-Saunders et de Beauharnois tuent chaque année $44 \%$ des anguilles en dévalaison migratoire. Cette situation est donc loin d'être naturelle et a un impact sur la pêche.

Les changements environnementaux causés par des politiques favorisant la croissance économique, les industries et le développement hydroélectrique ont contribué le plus au déclin des pêcheries. Toutefois, aucune mesure importante n'a été prise par les entreprises ou les États impliqués. Les gouvernements n'ont pas prévenu ou empêché la dégradation environnementale causée par ces industries et ont plutôt demandé, comme nous le verrons, aux pêcheurs de rationaliser leurs activités afin de protéger les anguilles et les esturgeons. Ce processus reflète une tendance plus large dans le contexte actuel marqué par les valeurs néolibérales qui favorise le développement capitaliste plutôt que la protection environnementale tout en délégant la responsabilité de la conservation aux utilisateurs.

Les anguilles argentées et les esturgeons noirs sont désormais soumis à des statuts de conservation. En plus des traités internationaux et des conventions pour protéger ces ressources, les anguilles sont maintenant désignées au Canada par le Comité sur la situation des espèces en péril au Canada (COSEPAC) comme une espèce en danger ${ }^{3}$. Cela rend possible certaines mesures de protection et de mitigation et l'étude de l'évolution des populations. Plus particulièrement, Hydro-Québec a dû installer quatre échelles de montaison pour les civelles d'anguilles, montrant fièrement sa bonne volonté environnementale, c'est bien peu par rapport à ses 5200 barrages; ironiquement, l'anguille est la mascotte de l'entreprise. Cette dernière tâche aussi de capturer quelques adultes en amont de Beauharnois afin de leur permettre d'éviter les turbines létales. La portée de ces mesures peut sembler insuffisante compte tenu de l'ampleur des moyens et des infrastructures de l'entreprise.

28 En ce qui concerne l'esturgeon noir, il est déjà reconnu en tant qu'espèce en danger de disparition internationalement et le COSEPAC veut en faire de même pour la population du Québec, qui est actuellement menacée, un statut moins précaire. Ce diagnostic est vivement contesté par les pêcheurs et les chercheurs du Québec qui gèrent étroitement cette population depuis plus de 20 ans. En effet, les contraintes imposées depuis les années 1990 sur les prises montrent maintenant leurs effets positifs et des signes de bonne santé de l'esturgeon noir sont indéniables dans le fleuve et son estuaire. Cependant, le COSEPAC, une agence fédérale, ne prend pas en compte ces recommandations, pas plus que les rapports des ministères provinciaux et des pêcheurs dans la mise en place de ces statuts. Les pêcheurs québécois déplorent par ailleurs qu'ils n'aient jamais vu un chercheur de cette agence sur leur territoire et ils interrogent la validité et la légitimité de leurs savoirs sur l'espèce.

29 Le statut précaire des pêches d'anguilles et d'esturgeons est lié à des causes politiques et au contexte historique du développement économique provincial, fédéral et international marqué par l'industrialisation et les idéologies modernistes qui ont contribué à détériorer l'intégrité et la vigueur de l'environnement du St-Laurent. 
Différentes études anthropologiques se sont penchées sur les pêches au Québec. Les premières ont été réalisées dès les années 1930 par les étudiants de Robert Redfield de l'Université de Chicago ; un de ces étudiants, Horace Miner a étudié le village de St-Denis de Kamouraska. Les études rurales en sciences sociales au Québec ont commencé dans la deuxième moitié du XIX ${ }^{\mathrm{e}}$ siècle par Léon Gérin puis par Gérald Fortin au Xxe siècle. Plus particulièrement, les premières études sociologiques et anthropologiques spécifiques aux communautés côtières ont été réalisées à l'Université Laval dans les années 1950 sous la direction de Marc-Adélard Tremblay. Dans les années 1960, ce dernier a dirigé un groupe d'étudiants qui, pour plusieurs, sont à leur tour devenus professeurs à Laval. Plusieurs études approfondies de communautés ont été faites sur la Basse-côte nord, notamment par Yvan Breton (1995), Paul Charest (1973) et Pierre Beaucage (1968). Ils ont examiné les activités de pêche, les structures sociales et l'organisation économique de différents villages dans les contextes québécois et canadien, et ils ont décrit les facettes multiples de la marginalisation de ces groupes. D'autres études menées par Claude Bariteau et Aliette Geistdoerfer ont examiné les Îles de la Madeleine. Certaines études de suivi ont été complétées dans les années 1990, sans qu'elles ne s'inscrivent dans un programme de recherche systématique. Les pêches d'anguilles et d'esturgeons n'ont pas fait l'objet de beaucoup d'attention en anthropologie au Québec. Un mémoire de maîtrise en anthropologie (Gilbert 1995) examine la marginalisation économique et la différenciation interne des pêcheurs et un mémoire de géographie (Levesque 2014) examine cette pêche et l'identité régionale. En sciences naturelles, des études des gouvernements fédéral et provincial ont examiné le déclin des ressources et l'impact des contaminants et des barrages hydroélectriques sur la mortalité (Verreault et al. 2012), une équipe de biologiste de l'Université Laval étudie la reproduction des anguilles et un biologiste à Rivière-duLoup travaille actuellement en collaboration avec les pêcheurs locaux pour poursuivre ces recherches.

\section{La pêche à l'anguille}

31 La pêche à l'anguille se fonde sur une technique avérée qui a peu changé historiquement et qui a été éprouvée grâce à la pêche aux bélugas. Dans l'estran des eaux saumâtres de l'estuaire, les pêcheurs installent leur filet fixe, la fascine. Ce filet de sept mètres de hauteur par 40 à 700 brasses de longueur, est posé face à la propriété terrienne des pêcheurs. Le mur de filet que constitue la fascine interrompt la dévalaison migratoire des aguilles à l'automne. Elles sont capturées dans des coffres installés à l'extrémité des filets qui sont relevés lors de chaque marée basse pendant la saison de pêche, de septembre à novembre. En plus des droits de propriété aux sites de pêche, l'équipement nécessaire pour réaliser cette pêche coûte environ 200000 \$, et plusieurs disent avoir besoin en plus d'environ 5000 \$ chaque année pour assurer leurs activités. Les pêcheurs sont au nombre de deux à quatre par équipe et appartiennent généralement à la même famille (frères, neveux, père et fils). La pêche aux anguilles occupe 20 semaines par années, au moment de la pêche elle-même ainsi que lors de l'installation des équipements au printemps et à l'été et lors de leur entretien avant l'hiver et l'apparition des glaces sur le fleuve. Un aspect important de cette pêche est qu'elle est étroitement liée aux droits de propriété et aux activités terrestres des pêcheurs. En effet, elle dépend directement d'un accès fiable au fleuve et pour cette raison, elle a été menée par des fermiers dont une des extrémités de leur propriété est limitrophe de l'estran, suivant le patron de distribution des terres instauré par la colonie française au moment de l'établissement des seigneuries. Cette 
pêche est réalisée en complément des autres travaux fermiers, dont l'agriculture, l'élevage et la coupe de bois.

Depuis l'arrivée des premiers colons, l'anguille a été pêchée pour la consommation locale, la vente dans les autres villages et plus tard pour l'exportation. Dès les années 1920, avec la venue du transport sur rails, elles étaient notamment acheminées aux États-Unis par train, vivantes sur la glace. Jusqu'aux années 1960, les prix demeurèrent bas, de sorte que le nombre de pêcheurs n'a pas augmenté pendant cette période. Le gouvernement n'avait alors pas encore établi de mesures de gestion de ces pêches. Dans les années 1950, les prix augmentèrent peu à peu, passant de neuf cents à un dollar le kilogramme en 1960, puis à cinq dollars à la fin des années 1960. Ces augmentations sont liées à l'exportation croissante des anguilles en Allemagne et au Pays-Bas. L'anguille, qui était plutôt une ressource de subsistance, est alors devenue commercialement lucrative. À partir des années 1960, les ventes étaient principalement vouées à l'exportation.

Face à ce contexte favorable, le gouvernement décida de donner des permis de pêche à tous ceux qui en faisaient la demande afin de faire profiter le plus grand nombre de cette source de revenu très intéressante dans cette région peu prospère. Bientôt, la région accueillit plus de 1000 pêcheurs actifs. Le but du gouvernement était d'augmenter les revenus des habitants de la région et de promouvoir sa modernisation. Cela a cependant mené à l'intensification non contrôlée de l'effort de pêche.

Dans les 1960, guidé par le programme fédéral du bureau d'aménagement de l'Est du Québec, le gouvernement du Québec a instauré une stratégie concernant les pêches artisanales, recommandant la fermeture de toutes les pêcheries « non productives » et de faible valeur commerciale. Les anguilles échappent au couperet en raison des revenus importants qu'elles génèrent. En 1974, le gouvernement paie même des pêcheurs pour voyager en Scandinavie afin d'y apprendre la maîtrise de techniques plus efficaces. Il a également payé pour la mise à neuf des équipements de pêche et a employé des techniciens pour installer de nouveaux équipements.

Pendant cette période d'abondance de ressources et de revenus, les pêcheurs ont bénéficié de bons prix, mais ils ne contrôlaient aucun aspect de la commercialisation, qui était déléguée entièrement à un intermédiaire qui achetait directement et individuellement aux pêcheurs leur production et la revendait dans ses réseaux. Cette exclusion des pêcheurs du processus de commercialisation a été exacerbée par leur éloignement physique des marchés centraux de Montréal, de Toronto et de la côte est américaine et par leur unilinguisme français.

Dans les années 1970, des études montrent un très fort taux de contamination des anguilles aux biphényles polychlorés et leur exportation vers l'Europe a été immédiatement suspendue. Bien que l'innocuité du produit ait été rétablie les ventes sur le marché international n'ont ensuite jamais repris. Le faible volume pêché depuis les années 1990, lié au déclin drastique des populations au Québec, ne le permettrait plus de toute façon.

Les préoccupations gouvernementales concernant la dégradation des stocks d'anguilles au Québec ont été limitées jusque dans les années 1990 à un point tel que ce sont les pêcheurs eux-mêmes qui, par le biais de leur association, ont demandé une rencontre avec des biologistes du gouvernement du Québec pour les informer du déclin alarmant des populations d'anguilles relevées dans leurs fascines. L'association a été très proactive et a cherché des solutions pour reconstruire les stocks. Elle a notamment instauré un 
programme d'ensemencement, en collaboration avec le gouvernement, permettant de relâcher dans un lac de la région 40000 civelles, achetées au Nouveau-Brunswick. Un des biologistes soutient qu'avant que les pêcheurs n'interviennent, personne au gouvernement ne connaissait cette situation et que sans leur action, les anguilles auraient probablement complètement disparu de l'estuaire. De ces premières collaborations ont émergé de nombreuses autres initiatives (Doyon 2015), ayant influencé notamment les mesures de mitigations instaurées par Hydro-Québec sur ses barrages hydroélectriques (MRNF 2009).

Une mesure majeure de gestion des populations d'anguilles a été de racheter aux pêcheurs leurs permis de pêche en vue de diminuer de moitié les prises, avec pour objectif une diminution de 10 à $20 \%$ du taux de mortalité ${ }^{4}$. Pour y arriver, le gouvernement a, en 2009, financé le rachat volontaire des permis de pêche. Le taux de compensation était de 5000 \$ par site de pêche auquel s'ajoutait un pourcentage des revenus de la pêche des trois années précédant le rachat. La compensation par pêcheur pouvait s'élever à plus de $100000 \$$ par pêcheur pour un montant total de compensations de 2,5 millions. Les pêcheurs qui vendirent leur permis ont perdu de façon définitive leurs droits d'accès de pêche sur la côte, un droit légué de génération en génération depuis l'arrivée des premiers colons et la pratique de la pêche aux bélugas. Ce programme a permis le rachat de 46 des 67 permis qui restaient dans l'Estuaire ${ }^{5}$. Les plus vieux pêcheurs et ceux qui n'avaient personne pour reprendre leur pêche ont notamment pris avantage de ce programme.

Le programme de rachat a été appuyé par certains pêcheurs actifs, qui y ont vu l'opportunité de diminuer le nombre de pêches et conséquemment d'augmenter leurs propres captures. Des vingt permis qui demeurent actifs, seulement quinze sont effectivement pêchés ${ }^{6}$. Malgré ces nombres réduits, la production totale a continué de diminuer pour atteindre un creux sans précédent de 14 tonnes en 2013. Ceux qui restent dans la pêche sont des producteurs laitiers et des propriétaires de poissonneries. Ils disent qu'ils continuent de pratiquer la pêche car ils sont des vrais pêcheurs, ceux qui l'ont fait depuis des générations et ceux qui veulent léguer la pêche à leurs enfants. Ils revendiquent leurs droits et leur légitimité à le faire.

Malgré le déclin drastique des anguilles, la fermeture virtuelle de ces pêches est passée relativement inaperçue, et les causes principales ne sont pas abordées dans le débat public. La gestion des pêches d'anguilles a suivi les logiques néolibérales de la privatisation et une rationalité du tout pour le profit. En effet, les politiques de gestion proposent de nouvelles régulations qui modifient la tenure foncière et l'accès aux ressources naturelles. La fermeture temporaire des turbines de barrages hydroélectriques ou la multiplication de passes migratoires sur ces mêmes barrages n'ont pour leur part pas été envisagées, les politiques se concentrant plutôt sur les actions individuelles d'un petit groupe d'acteurs aux intérêts divers. Les pêcheurs se sont, volontairement et graduellement, retirés de la pêche, contribuant à rendre invisible cette situation pour la population en général. 
Figure 1 : Pêcheur d'anguilles au travail

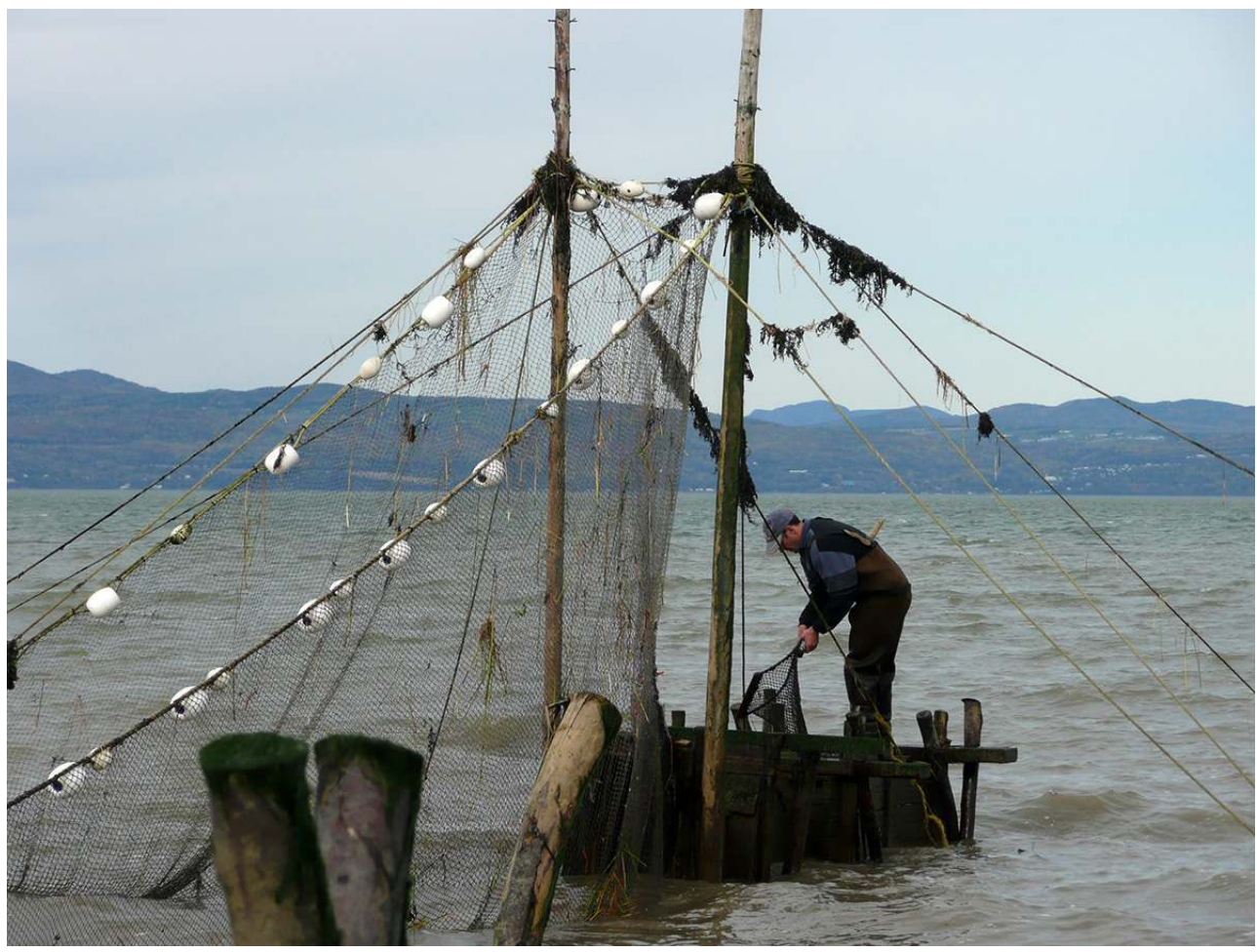

(c) S. Doyon

Figure 2 : Pêcheurs d'anguille au travail

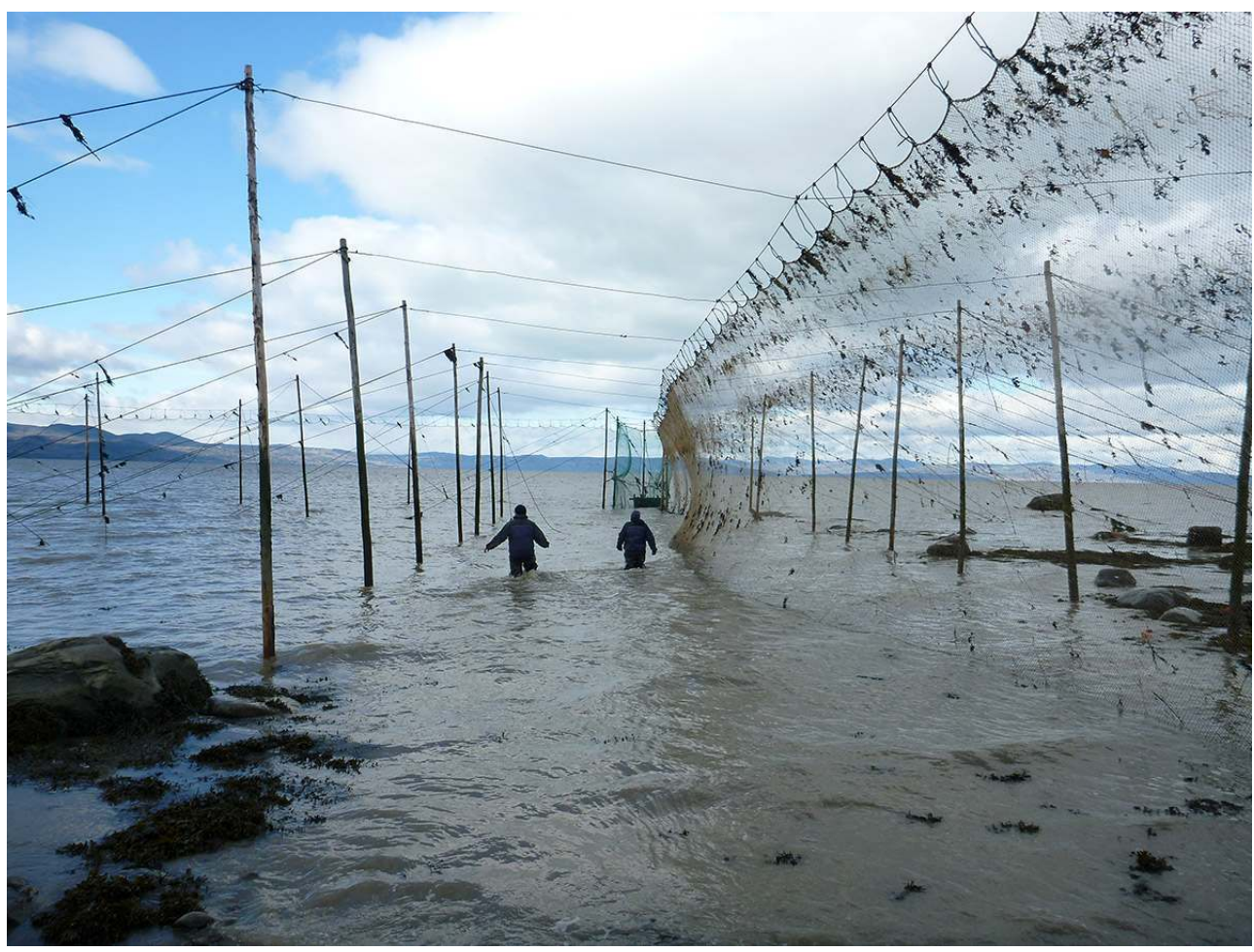

(c) S. Doyon 


\section{L'esturgeon noir}

41 La pêche de l'esturgeon noir a, quant à elle, une histoire un peu différente, mais elle a conduit elle aussi à l'instauration d'une gestion de la ressource en collaboration avec les pêcheurs qui culmine par une forme de privatisation: l'attribution de quotas de pêche. Cette espèce avait complètement disparu du fleuve de 1967 à 1978. Au milieu des années 1980, les pêcheurs d'anguilles ont vu réapparaître les esturgeons noirs dans leurs fascines, où ils étaient pêchés traditionnellement. Les pêcheurs ont alors entrepris de profiter de cette nouvelle manne au moment où les captures d'anguilles étaient en déclin.

42 Alors que l'esturgeon noir avait été pêché à gué dans le fleuve, au tournant des années 1980-90, certains pêcheurs d'anguilles, grâce à un capital amassé notamment avec la pêche à l'anguille et du fait d'une expérience familiale particulière dans le transport maritime (croisiéristes), ont entrepris de se munir de bateaux afin d'aller à la rencontre de l'esturgeon au large et d'y tendre des filets pour les capturer. Cette stratégie s'est avérée extrêmement lucrative pour ces premiers audacieux et, rapidement, les autres pêcheurs les ont rejoints dans cette nouvelle pratique de pêche. En quelques années, le moyen estuaire fut traversé par de petites embarcations de six mètres des mois de juin à septembre : une nouvelle petite pêche commerciale voyait le jour.

43 Cette nouvelle pêche au large a favorisé l'émergence de pratiques nouvelles qui ont une incidence sur les rapports sociaux et la gestion des pêches. Les pêcheurs à gué d'esturgeons noirs ont adapté leurs savoirs de la pêche et de la mer à une pratique au large. Ils ont dû tout apprendre, n'ayant même jamais auparavant possédé de bateaux. Les récits qu'ils nous en font témoignent de la forte impression que la pêche leur laisse et de la peur qu'ils ont de la mer encore aujourd'hui après plus de vingt ans de pratique. Ils soulignent à plusieurs reprises les risques qu'ils encourent et le caractère très changeant du fleuve qui les a mis souvent dans des positions périlleuses. Les courants, les îles, les saisons, les gels ont dû être appris et apprivoisés par les nouveaux pêcheurs en mer, des dimensions qui, bien qu'importantes pour la pêche à gué, diffèrent complètement une fois en mer.

44 L'abondance de la pêche au large a certes stimulé les pêcheurs à gué pour se convertir, mais cette conversion est due également à l'impératif de défendre et de faire respecter son territoire. En effet, les pêcheurs les plus téméraires et avec le plus de savoirs en navigation ont osé aller toujours plus loin pour poser leurs filets, empiétant sur les territoires informels traditionnels des autres pêcheurs qui continuaient leurs pratiques à gué. Des pêcheurs, traditionnellement à gué, qui ne s'étaient jamais côtoyés, chacun étant sur son bout de terre, ont été amenés à le faire et ont saisi la finitude du fleuve et de ses ressources. La mer, qui était auparavant simplement marquée par des repères terrestres (les terres agricoles qui viennent s'échoir dans la mer et les îles qui s'égrènent face à ces dernières), devient un espace disputé entre pêcheurs qui, n'étant plus clairement déterminés par leur appartenance à la terre, eurent à s'aventurer et à s'approprier ce que d'aucuns jugent qui leur revient de droit. Certains parmi eux pensent que le fleuve se donne à ceux qui le comprennent et le défient et que la recherche de bons sites de pêche dans cet espace houleux revient au plus téméraire et au plus connaissant. Dans la foulée, ils ont constaté les quantités importantes d'esturgeons noirs capturées, étonnement beaucoup plus élevées que celles pêchées à gué. Certains pêcheurs ont alors sonné 
l'alarme préventivement, menant les responsables gouvernementaux à encadrer cette nouvelle intensité de prélèvement.

La gestion de la pêche à l'esturgeon noir a été établie sur les récents savoirs et pratiques développés par les pêcheurs en mer. Afin de réglementer la pratique, ce sont les pêcheurs eux-mêmes qui ont proposé les délimitations des zones de pêches exclusives à partir de leurs connaissances de la mer. Les zones correspondent grosso modo aux lieux de vie des pêcheurs qui pratiquent ces pêches. Le savoir plus approfondi des eaux possédé par certains, du fait de leurs expériences en mer pour le transport maritime, a-t-il pu influencer d'une façon ou d'une autre la désignation de ces zones de pêche? Quoiqu'il en soit, il semble s'être établi un certain déséquilibre dans la capacité de captures entre les divers pêcheurs : pour chacune des zones exclusives de pêche, les pêcheurs se sont vus accorder des longueurs de filets déterminées en fonction de leurs demandes. Certains ont demandé des milliers de brasses de filet de pêche. D'autres sont demeurés modestes dans leur demande, ne sachant pas quel était l'objectif du gouvernement et croyant que la situation était sous étude ; un pêcheur explique la situation en disant : «j'en ai demandé seulement 300 pieds, je ne voulais pas être extravagant ». Ces attributions sont cependant demeurées et ce même si le pêcheur dit que s'il avait su, il en aurait demandé beaucoup plus et que désormais il est désavantagé par rapport à d'autres en raison de sa sobriété initiale.

La gestion de l'esturgeon noir par les autorités s'est poursuivie par l'attribution d'un quota par pêcheur en fonction de la longueur de filet auquel celui-ci a droit. En plus d'un nombre annuel fixe de poissons, les pêcheurs doivent, sur la base de mensurations réglementées, remettre à l'eau les gros géniteurs ainsi que les juvéniles. Malgré certaines réticences initiales, les pêcheurs collaborent pleinement et ils affirment que ces mesures ont permis d'assurer la santé des stocks de cette espèce très lucrative dont la commercialisation est cependant menacée du fait de la précarité du statut de l'espèce à l'échelle internationale.

Les pêches à l'anguille et à l'esturgeon noir sont associées à la fois par la technique de pêche qui a été utilisée pour les capturer et par une histoire environnementale et une écologie politique qui en a exacerbé l'exploitation. La pêche à l'anguille et à l'esturgeon, et le travail concret qu'elle suppose, sont également étroitement chevillés à la gestion traditionnelle et contemporaine qui en est faite et aux principes de conservation qui les ont guidés. Par ailleurs, ce tissu unissant travail, gestion et conservation est lui-même coloré par l'accès et le contrôle des espaces de pêche et par les moyens de travail. Le contexte mondial actuel, dans lequel prévalent des principes néolibéraux pour l'utilisation de la nature, tels que la privatisation, la dérégulation et la responsabilisation individuelle des pratiques, encadre désormais ces pêches.

\section{Marchandisation des anguilles et des esturgeons noirs et valorisation du patrimoine local}

La mise en marché des anguilles et des esturgeons noirs est rendue précaire par la diminution des captures de ces espèces et par la diminution de l'intensité des activités de pêche. Mais l'examen de son évolution révèle également d'autres dimensions qui ont influencé son histoire, mais qui intègrent toutefois les enjeux de protection de ces ressources. 
49 En ce qui concerne les anguilles, la vente se concentre depuis les années 1980 sur le marché canadien, plus particulièrement les marchés «ethniques » de Montréal et de Toronto. Dans le marché québécois, les ventes n'ont pas repris après les années 1960. Avec la Révolution tranquille, les familles ont d'une part peu à peu cessé leurs pratiques de jeûne carné les vendredis, les « vendredis maigres ", et, d'autre part, d'autres types de poissons, comme les salmonidés et des poissons blancs génériques faciles à cuisiner et au goût peu prononcé, ont envahi les marchés, volant la vedette à ces bêtes « impossibles à tuer » selon les cuisinières ménagères canadiennes-françaises.

Jusqu'à il y a environ trois ans, tous les pêcheurs continuaient de vendre leurs captures à un seul acheteur. Pendant longtemps cette situation leur a convenu, l'acheteur se déplaçait au domicile de chacun des pêcheurs pour récupérer les prises et ces derniers n'avaient pas eux-mêmes à chercher des débouchés, une tâche complexe et énergivore s'ajoutant à leurs nombreuses activités journalières liées au travail de la ferme.

51 Par ailleurs, les pêcheurs n'ont pas, historiquement, voulu se regrouper pour structurer la mise sur le marché. Ils ont longtemps refusé de collaborer à cette étape du processus par manque de temps pour établir une procédure, mais aussi car ils sont scrupuleux de ne pas révéler leurs statistiques de captures, révélatrices de leur revenu. Ils ont aussi longtemps pensé tirer un meilleur profit en négociant individuellement avec leur acheteur et en consolidant leur lien personnel avec lui plutôt qu'en collectivisant la mise sur le marché.

Les jeunes pêcheurs d'aujourd'hui semblent toutefois vouloir changer cette situation. Ils sont insatisfaits du système organisé autour d'un acheteur unique. Ce dernier emploie d'ailleurs des tactiques de négoce pour lesquelles la plupart ne sont pas dupes. En effet, bien qu'il recueille la marchandise directement auprès des pêcheurs, il ne remplit pas toujours sa part de l'échange selon les « règles du jeu » et ne paie pas immédiatement la marchandise qu'il convoite. Les pêcheurs relatent qu'il emploie différents subterfuges afin de différer les paiements, comme prétendre qu'il a oublié l'argent chez lui et qu'il paiera la prochaine fois, ou bien qu'il a des problèmes d'argent à la suite d'un bris sur son camion et qu'il ne peut offrir de meilleurs prix. Les pêcheurs sont conscients de leur situation précaire, savent qu'ils sont dépendants de cet unique lien de vente, et ils se demandent chaque automne si cet acheteur vieillissant se présentera.

Certains poissonniers tentent désormais de renverser le seul acheteur d'anguilles et de le remplacer en offrant de meilleurs prix aux pêcheurs du coin afin d'assurer leur approvisionnement, mais la confiance de ces derniers est difficile à acquérir. D'autres tentent de développer un réseau de vente auprès des acheteurs chinois de Toronto, un réseau que contrôlait jusqu'à présent l'intermédiaire exclusif. Plusieurs barrières doivent être levées en plus de celle de la langue, comme la consolidation d'équipements pour la conservation et le transport sur une longue distance (plus de $1000 \mathrm{~km}$ ) des anguilles vivantes et la compréhension des règles non écrites des transactions dans le cadre d'une autre culture d'affaire, un enjeu dont Tsing (2015) discute notamment de façon éloquente avec le cas des champignons matsutake, et qui est loin d'être évidente.

Pour ce qui a trait à l'esturgeon, les pêcheurs sont contraints de les vendre localement uniquement, car le statut d'espèce en péril ne permet pas la vente à l'extérieur de la frontière provinciale. De même, les faibles quantités pêchées ne permettent pas la commercialisation dans un grand nombre de points de vente, et les poissonneries locales tentent le plus possible d'assurer leur approvisionnement en établissant des ententes avec les pêcheurs. Les pêcheurs doivent également distinguer leur produit de l'esturgeon 
d'aquaculture, provenant d'une sous-espèce différente, mais dont la commercialisation mise sur l'attrait historique de l'esturgeon noir sauvage.

\section{La transformation et les nouveaux marchés} saucisses de poisson et d'autres produits transformés qu'ils vendent dans des marchés de poissons, des épiceries fines et des supermarchés de la région du Kamouraska et ailleurs au Québec. Ils bénéficient aussi de l'arrivée de touristes estivaux friands de ces produits «du terroir» disponibles dans les commerces locaux. Le profit sur les produits transformés est important. Alors que, par exemple, l'anguille vivante est vendue à $8 \$$ le kilogramme, son prix peut atteindre, une fois transformée, entre 55-85 \$ le kilogramme. Le processus de transformation n'est pas simple et il est très contrôlé. Le ministère de l'Agriculture, des Pêcheries et de l'Alimentation exige de nombreuses conditions pour l'élaboration de produits transformés et l'émission de permis de vente. Ces pêcheurs et leur famille doivent investir beaucoup de temps, d'argent et d'énergie pour y parvenir en prenant des cours de cuisine et de mise en marché, en louant un espace pour transformer leurs captures, en s'enregistrant en tant que compagnie et en investissant du temps pour présenter leur produit dans des festivals et des évènements promotionnels, généralement éloignés de leur lieu de résidence.

Ces activités représentent un temps considérable et un investissement personnel important, difficile à conjuguer avec les activités de la ferme et la vie familiale. Certains se sont associés pour mutualiser les coûts et les résultats sont encourageants. En plus de ventes dans des commerces, ils tentent aussi de développer des collaborations avec des chefs vedettes dans certains restaurants qui favorisent les menus saisonniers à base de produits québécois distinctifs. Cependant, la compétition est féroce dans le marché de niche du terroir et bien qu'il y ait un véritable intérêt pour les produits locaux, les pêcheurs trouvent ardu de mettre en marché leur produit dans des épiceries éloignées où ils ne peuvent pas faire de suivi direct sur les ventes et la mise en étalage. 


\section{L'Indication géographique protégée}

59 L'Association des pêcheurs d'anguilles et d'esturgeons s'est engagée en 2014 dans des démarches afin de développer un statut d'indication géographique protégée de ces espèces, à la suite d'une sollicitation du ministère de l'Agriculture, des Pêcheries et de l'Alimentation' ${ }^{7}$. Une IGP peut être reconnue si un produit est spécifique et qu'il se distingue d'un produit de masse, que l'origine du produit peut être retracée et clairement identifiée, qu'il ne peut être reproduit ailleurs et qu'il soit original ; des caractéristiques qui s'appliquent clairement aux anguilles et aux esturgeons de l'estuaire du St-Laurent.

L'IGP est pensée par les promoteurs du gouvernement comme un outil de développement, qui met en avant la dimension artisanale d'un produit, la notion de patrimoine et l'économie du terroir. En faisant la promotion d'un produit par le bais d'une IGP, les responsables nous ont précisé qu'ils visaient notamment le développement régional et économique tout en moussant la popularité du produit. L'IGP permet, selon eux, de redonner de la valeur à ce qui en avait perdu et d'offrir une visibilité à des produits, mais elle ne constitue pas un outil permettant d'augmenter les ventes à court ou moyen terme.

61 Par le biais de l'IGP, les pêcheurs souhaitent bien sûr faire connaître leur produit au Québec et ailleurs dans le monde. Ils espèrent également que cette démarche permette d'éduquer la population sur ces espèces mal aimées et de les valoriser. De plus, les pêcheurs ont une autre attente face à l'IGP, celle de protéger la ressource, comme ils nous l'ont tous répété. Ils veulent d'une part la protéger des politiques de gestion et de conservation du gouvernement fédéral qu'ils décrivent comme voulant «fermer ces pêches ", notamment par des politiques du COSEPAC qui visent à resserrer leur statut de conservation en rendant l'esturgeon noir "espèce en voie d'extinction » et l'anguille argentée « espèce menacée ». D'autre part, ils veulent que l'IGP protège la spécificité de l'esturgeon de l'estuaire face à la menace que représente la commercialisation d'esturgeons à museau court (Acipenser brevirostrum) et d'hybrides dans les Maritimes ainsi que l'exploitation exponentielle des civelles dans les Maritimes et sur la côte de la Nouvelle-Angleterre.

62 En effet, les pêcheurs d'esturgeons se voient confrontés au développement de l'aquaculture de l'esturgeon noir au Nouveau-Brunswick pour le caviar. Cet élevage mène à la vente de la chair des mâles, inutiles pour le caviar, sur le marché québécois et canadien, selon une stratégie de mise en marché qualifiant ce poisson « d'écologique et durable ", bien qu'il soit issu d'un prélèvement dans la population de la rivière St-Jean (NB). Pour les pêcheurs d'esturgeons noirs du Québec, ce n'est pas un hasard si l'interdiction de pêche qui les menace (en raison d'une éventuelle prochaine révision du statut de l'espèce de "menacée " à "en voie d'extinction») coïncide avec le moment où un nouveau marché d'esturgeon d'élevage dans les Maritimes, issu de longues et coûteuses années de recherche et de développement, est arrivé à une phase rentable d'exploitation. Déjà, cette récente capacité de production aquacole sature les grandes surfaces d'alimentation en chair d'esturgeons, alors que celui du Québec n'arrive pas à le pénétrer. L'IGP des esturgeons noirs de l'estuaire permettrait aux pêcheurs de faire reconnaître leur travail et d'éviter que soient confondus sur les marchés les esturgeons d'élevage des Maritimes avec ceux sauvages du Québec.

Quant aux civelles, elles sont l'objet d'une explosion de leur pêche dans les Maritimes et sur la côte est des États-Unis dont la production est destinée au marché international'. 
Contrairement aux difficultés de commercialisation de l'anguille argentée dans l'estuaire, le prix des civelles peut atteindre 5000 \$ le kilogramme, ce qui suscite un engouement tel que les pêcheurs de l'estuaire craignent pour la reproduction de l'espèce. Ils croient que l'IGP pourrait leur donner un levier supplémentaire pour les protéger d'une éventuelle surexploitation.

Le mécanisme de mise en place d'une IGP dépend d'un responsable au sein du ministère et est encadré par un processus organisé et fermé. La première étape est de réaliser une étude de cas, ce qui a été complété en 2015 par une firme de consultants indépendants du gouvernement présentant les résultats d'une enquête réalisée auprès des pêcheurs sur les pratiques de pêche et la commercialisation de leurs produits (Jacques et al. 2015). Après cette première étape, le projet doit être accepté par le ministère pour être repris par le Conseil sur les appellations réservées et les termes valorisants (Cartv), un organisme indépendant du ministère, qui doit le reconnaitre et qui s'engage à son tour dans le processus.

Si cette planification peut sembler simple et directe sur papier, dans les faits, les processus sont longs (au moins trois ans) et incorporent plusieurs enjeux complexes. Notamment, le processus de désignation d'une IGP halieutique fait face à des réalités de production hétérogènes, difficilement commensurables et qui ne correspondent pas toujours aux normes bureaucratiques établies pour baliser la désignation des appellations réservées et des IGP agricoles. Par exemple, le cidre de glace ou l'agneau appartiennent à deux univers agricoles très contrastés avec des caractéristiques qui se comparent difficilement entre eux et avec l'anguille et l'esturgeon. Ce sont des produits qui relèvent de procès de travail, de production et de transformation qui peuvent difficilement être encadrés par des critères identiques. Il en va de même pour les organisations professionnelles avec lesquelles le ministère doit travailler, qui sont hétérogènes, constituées d'intérêts variés et de perspectives différentes.

Cette hétérogénéité rend la situation complexe pour les décideurs, restant dans le flou en ce qui concerne l'avenir de la structuration de l'IGP envisagée par les pêcheurs de l'estuaire. Toutefois, comme nous l'ont précisé des répondants, la question du cahier des charges, qui constitue généralement un problème pour les aspirants à une IGP, ne serait pas un problème dans le cas de l'anguille et de l'esturgeon, car les pratiques de pêche sont encadrées par des techniques de gestion strictes depuis des décennies. Quant à la traçabilité, elle est claire car la présence des espèces est très localisée. Ces aspects rendent le processus déjà plus simple que dans d'autres cas, comme le vin de glace, où tous les producteurs n'employaient pas les mêmes techniques de production, influençant la qualité et l'uniformité. Ainsi, bien que les pêcheurs se soient investis dans cette démarche et qu'ils aient posé les actions nécessaires pour lancer l'initiative, il semble que le dossier n'ait pas bougé du côté de l'administration ministérielle.

À cette situation s'ajoutent les coûts très élevés qu'implique le développement d'une IGP et qui doivent être assumés par l'Association des pêcheurs et les transformateurs. Comme le spécifie le rapport, le cahier des charges, le plan de contrôle et les frais annuels pour les deux espèces s'élèveront à plus de $93000 \$$ (Jacques et al. 2015). Ces frais sont très élevés en comparaison des revenus que procure leur pêche et si l'on considère le caractère incertain et imprévisible de leurs captures. De plus la mutualisation des frais n'est pas évidente compte tenu du caractère indépendant des pêcheurs relativement aux questions de vente. 
68 Au même moment que ces mesures de mise en marché sont entreprises, il y a depuis quelques années un intérêt renouvelé pour ces pratiques en tant qu'elles incarnent un patrimoine culturel distinctif. Des nouvelles dans les journaux, des documentaires, des émissions de radio spécialisées, des programmes de télévision, des émissions de cuisine se dédient à ces espèces et à l'art de les cuisiner, rendant hommage aux racines canadiennes-françaises de ces aliments. En plus de cet intérêt médiatique, des initiatives pour faire la promotion de ces pêches prennent place dans les régions, des expositions de photos (ambulantes en 2012-2013) ont présenté l'art de la pêche à la fascine, un film documentaire, un festival à Charlevoix (même si on n'y pêche plus depuis des décennies) et un musée privé de l'anguille, géré par une retraitée de la pêche, est ouvert au public l'été. Finalement, l'équipement de la pêche est promu comme dimension patrimoniale du paysage côtier de la région et est mis en évidence sur les routes écotouristiques du Bas-StLaurent. Ces initiatives insistent sur le caractère traditionnel de la pêche, qui plaît aux touristes, mais occultent les enjeux contemporains auxquels les pêcheurs font face.

69 La récupération patrimoniale des pêches commerciales du fleuve est intéressante d'un point de vue culturel et muséal. Toutefois, les pêcheurs ne voient pas nécessairement de retombées économiques de ces différentes initiatives qui les mettent en avant. Il semble plutôt que l'imaginaire auquel renvoient ces initiatives patrimoniales met en valeur un fleuve de loisir et de beauté et non pas un lieu de pratiques, d'exploitation et de travail, qui cadre de moins en moins avec les idéaux conversationnistes qui inondent la région.

70 Ainsi, la marchandisation des anguilles et des esturgeons ne va pas de soi historiquement et les démarches récentes impliquant une IGP ne sont pas sans contradictions et défis, notamment quant à la prise en considération des particularités des systèmes socioécologiques de production locale et la rigidité organisationnelle et normative des processus de certification face aux situations économiques diversifiées des producteurs (Doyon à paraître). Le succès d'une IGP dépend notamment d'une compréhension fine du contexte socio-environnemental et de la prise en compte des particularités de la production et de sa mise en marché. L'effort d'élaboration des IGP pour ces deux pêches phares de l'estuaire témoigne de ce que soulignent Bérard et Marchenay (2002): l'importance du mode de tenure, de la répartition des capitaux et des activités économiques entre les acteurs et de leur histoire, des aspects qui cadrent parfois difficilement avec le cadre normatif des IGP conventionnelles.

\section{Conclusion}

71 La commercialisation et la protection des bélugas, des anguilles et des esturgeons sont enchâssées dans l'écologie politique de ces pêches ainsi que dans les politiques de conservation et de gestion de ces espèces. Les enjeux de marchandisation sont directement liés à l'écologie de l'espèce, aux transformations environnementales du milieu, aux politiques gouvernementales de développement, à l'économie régionale, à la gestion étatique des ressources et aux politiques de conservation des espèces.

72 La pêche aux bélugas, anguilles et esturgeons s'ancre dans l'écologie régionale, l'économie mondiale et les cultures locales qui organisent ces pratiques. L'analyse de la pêche au béluga montre que le processus qui l'a marqué a, d'une part, déterminé l'évolution des pêches d'anguilles et d'esturgeons et, d'autre part, qu'il se situe en droite 
ligne des politiques économiques nationales de primarisation et du capitalisme international.

73 L'examen de l'exploitation des anguilles et des esturgeons montre également que les politiques de gestion et de mise en marché ont contribué à la marginalisation de ces activités et à un processus de différenciation socio-économique dans la région. Le processus de privatisation en cours des pratiques de pêche, par le rachat de permis d'anguilles et par l'attribution de quotas d'esturgeon, a des effets régionaux et crée différentes classes de pêcheurs, comme ce fut le cas dans d'autres contextes (Carothers \& Chambers 2012). Les politiques de gestion de ces pêches ont exercé une pression sur plusieurs pêcheurs et les plus marginaux ont dû abandonner, incapables de maintenir cette pratique dans le contexte de la diminution brutale des stocks et des enjeux de mise en marché. Seuls ceux ayant les reins les plus solides (car héritiers des sites les plus productifs) et pouvant compter sur d'autres activités économiques lucratives (généralement l'agriculture ou la propriété de poissonneries) peuvent s'y maintenir. Certains pêcheurs ayant dû abandonner leurs propres pêches, pêchent désormais pour ceux qui possèdent assez de moyens pour les employer. En effet, les quotas d'esturgeons ont permis une forme de contractualisation de la pêche et les pêcheurs les plus marginalisés se voient dans l'obligation de pêcher les quotas des pêcheurs les plus avantagés, accentuant la différenciation socio-économique dans cette région éprouvée.

Ainsi, les récentes démarches de transformation, de certification et de mise en marché alternatives sont possibles car les pêcheurs ne dépendent pas uniquement de la pêche pour leur survie. Cette stratégie implique encore une fois que seuls les acteurs dont la sécurité financière est assurée peuvent s'investir dans ces démarches longues et coûteuses, marginalisant encore davantage les autres pêcheurs et ces pêches commerciales, dont la disparition se déroule de façon silencieuse, sans protestation. Les pêches ont été d'une valeur commerciale locale importante et ont toujours été des activités qui ont amené un revenu supplémentaire ou de la nourriture aux familles. Leur disparition ne laissera pas demain des centaines de familles à la rue ou toute une communauté en choc, mais la diversité culturelle, économique et sociale des régions côtières est amoindrie et la transmission d'un savoir-faire écologique unique est perdue. Ironiquement, la disparition survient alors que des activités qui en font la promotion avec celle $d u$ paysage rural comme une part importante du patrimoine et alors que la valorisation gastronomique de ces espèces est en cours.

\section{BIBLIOGRAPHIE}

Acheson J. M. 2011 - Coming up Empty: Management Failure of the New England Groundfishery. MAST 10 (1) : 57-86.

Acheson J. M. \& Gardner R. J. 2004 - Strategies, Conflict, and the Emergence of Territoriality: The Case of the Maine Lobster Industry. American Anthropologist 106 (2) : 296-307. 
Andreatta S. \& Parlier A. 2010 - The Political Ecology of Small-Scale Commercial Fishermen in Carteret County, North Carolina. Human organization 69 (2) : 180-191.

Bavington D. 2010 - Managed Annihilation: An Unnatural History of the Newfoundland Cod Collapse. Vancouver (BC), University of British Columbia Press, 224 p. (Nature, History, Society Series).

Beaucage P. 1968 - Technologie de la pêche au loup-marin sur la côte nord du Saint-Laurent. L'Homme 8 (3) : 96-125.

Bérard L. \& Marchenay P. 2002 - Un exemple de «non patrimonialisation » : la carpe en Dombes. Montpellier, Centre d'Études et de Documentation « Ressources des terroirs - Cultures, Usages, Sociétés » du CNRS (Centre national de la recherche scientifique).

Blaikie P. \& Brookfield H. 1987 - Land Degradation and Society. Londres, Methuen, 284 p.

Breton Y. 1995 - L'effet récurrent du capitalisme sur une communauté de pêcheurs : St. Paul's River, Basse-Côte-Nord. In : Trudel F., Charest P. \& Simonis Y. (Ed.), La construction de l'anthropologie québécoise. Mélanges offerts à Marc-Adélard Tremblay. Québec, Les presses de l'Université Laval : 413-428.

Brockington D. \& Duffy R. 2010 - Capitalism and conservation: the production and reproduction of biodiversity conservation. Antipode 42 (3) : 469-484.

Bryant R. L. \& Bailey S. 1997 - Third World Political Ecology. Londres, New York, Routledge, 256 p.

Caron F., Dumont P., Mailhot Y. \& Verreault G. 2007 - L'anguille au Québec, une situation préoccupante. Le Naturaliste Canadien 131 (1) : 59-66.

Carothers C. 2008 - Rationalized out: discourses and realities of fisheries privatization in Kodiak, Alaska. In : Lowe M. E. \& Carothers C. (Ed.), Enclosing the Fisheries: People, Places, and Power Symposium. Bethesda, American Fisheries Society : 55-74.

Carothers C. \& Chambers C. 2012 - Fisheries privatization and the remaking of fishery systems. Environment and Society: Advances in Research 3 (1) : 39-59.

Charest P. 1973 - Écologie culturelle de la Côte-Nord du golfe Saint-Laurent. In : Tremblay M.-A. \& Gold G. L. (Ed.), Communautés et culture. Éléments pour une ethnologie du Canada français. Montréal, Éditions HRW Ltée : 33-82.

Charest P., Girard C. \& Rodon T. (Ed.) 2012 - Les pêches des premières nations dans l'est du Québec. Québec, Presses de l'Université Laval, 384 p.

Davis R. 2014 - A Cod Forsaken Place?: Fishing in an Altered State in Newfoundland. Anthropological Quarterly 87 (3) : 695-726.

Davis R., Whalen J. \& Neis B. 2006 - From Orders to Borders: Toward a Sustainable Co-managed Lobster Fishery in Bonavista Bay, Newfoundland. Human Ecology 34 (6) : 851-867.

Doyon S. 2002 - The Structural Marginalization of Artisanal Fishing Communities: The Case of La Boquita. Anthropologica 44 (1) : 83-98.

Doyon S. 2015 - Losing ground: The marginalization of the St. Lawrence eel fisheries in Québec, Canada. Marine Policy $61: 331-338$.

Fortin J. C. \& Lechasseur A. (Ed.) 1993 - Histoire du Bas-Saint-Laurent. Québec, Institut québécois de recherche sur la culture, $860 \mathrm{p}$. 
Fraga J., Villalobos G., Doyon S. \& Garcia A. (Ed.) 2008 - Gobernanza Costera en México: Descentralización y Manejo Ambiental en la Península de Yucatán. Plaza y Valdes, Universidad Autónoma de Campeche, 296 p.

Franck A. 2008 - Les pêcheries de l'estuaire et la guerre au marsouin. L'Escale nautique 57 : 64-68.

Gilbert R. 1995 - Marginalité et continuité: le cas des pêcheurs d'anguilles du corridor fluvial du SaintLaurent. Québec, projet de mémoire, Département d'anthropologie de l'Université Laval.

Gouvernement du Canada 2015 - Revue statistique annuelle 2010-2011, Pêches et Océans Canada, [En ligne] URL : http://www.qc.dfo-mpo.gc.ca/peches-fisheries/statistiquesstatistics/2010-2011/index-fra.html. Consulté en 2016.

Gouvernement du Québec 2011 - Le Bas-Saint-Laurent et ses municipalités régionales de comté (MRC), Institut de la Statistique du Québec, [En ligne] URL : http://www.stat.gouv.qc.ca/ statistiques/profils/region_01/region_01_00.htm. Consulté en 2016.

Hébert K. 2015 - Enduring Capitalism: Instability, Precariousness, and Cycles of Change in an Alaskan Salmon Fishery. American Anthropologist 117 (1) : 32-46.

Innis H. 1956 - Essays in Canadian Economic History. Toronto, University of Toronto Press, 418 p. Jacques L.-S., Rivas A. \& Danyod G. 2015 - Perfectionnement des connaissances afin de recommander une démarche d'appellation réservée ou de terme valorisant pour l'anguille d'Amérique et l'esturgeon noir pêchés commercialement dans l'estuaire du Saint-Laurent. Pour l'Association des pêcheurs d'anguilles et de poissons d'eau douce du Québec, avec l'accompagnement de la Table Agroalimentaire de la Chaudière-Appalaches (TACA).

Levesque M. 2014 - La pêcherie d'anguille du Kamouraska : essor et déclin d'une culture et d'une ressource. Québec, projet de mémoire, Département de géographie de l'Université Laval.

Mansfield B. 2004 - Rules of Privatization: Contradictions in Neoliberal Regulation of North Pacific Fisheries. Annals of the Association of American Geographers 94 (3) : 565-584.

Mansfield B. 2010 - "Modern" industrial fisheries and the crisis of overfishing. In : Peet R., Robbins P. \& Watts M. J. (Ed.), Global Political Ecology. Londres, New York, Routledge : 55-74.

McCay B. J. 1995 - Social and Ecological Implications of ITQs: An Overview. Ocean and Coastal Management 28 (1-3) : 3-22.

Menzies C. R. 2012 - The Disturbed Environment: The Indigenous Cultivation of Salmon. In : Colombi B. J. \& Brooks J. F. (Ed.), Keystone Nations: Indigenous Peoples and Salmon across the North Pacific. Santa Fe, School for Advanced Research : 161-182.

MRNF (Ministère des Ressources naturelles et de la Faune) 2009 - Une nouvelle mesure pour assurer le renouvellement de l'anguille d'Amérique. Communiqué de presse, le 17 mars 2009, du Cabinet du ministre délégué aux Ressources naturelles et à la Faune.

Musée de la mémoire vivante 2009 - Le dernier pêcheur de marsouins, [En ligne] URL : http:// www.memoirevivante.org/SousOnglets/AfficheSousOnglet?SousOngletId=2. Consulté en octobre 2016.

Olson J. 2011 - Understanding and contextualizing social impacts from the privatization of fisheries: An overview. Ocean and Coastal Management 54 (5) : 353-363.

Pauly D. \& Zeller D. (Ed.) 2016 - Global Atlas of Marine Fisheries: A Critical Appraisal of Catches and Ecosystem Impacts. Washington (DC), Island Press, 520 p.

Peet R., Robbins P. \& Watts M. J. (Ed.) 2011 - Global political ecology. Londres, New York, Routledge, $450 \mathrm{p}$. 
Pinkerton E. 2013 - Alternatives to ITQs in equity-efficiency-effectiveness trade-offs: How the lay-up system spread effort in the BC halibut fishery. Marine Policy 42 : 5-13.

Robitaille J. A. \& Tremblay S. 1994 - Problématique de l'anguille d'Amérique (Anguilla rostrata) dans le réseau du Saint-Laurent. Québec, Ministère de l'Environnement et de la Faune, Direction de la faune et des habitats, Bibliothèque Nationale du Québec, Rapp. Tech. ix +70 p., dépôt légal - ISBN 2-550-29181-6.

Verreault G., Mingelbier M. \& Dumont P. 2012 - Spawning migration of American eel Anguila rostrata from pristine (1843-1872) to contemporary (1963-1990) periods in the St. Lawrence Estuary, Canada. Journal of Fish Biology 81 (2) : 387-407.

Verreault G. \& Trencia G. 2011 - Atlantic Sturgeon (Acipenser oxyrinchus oxyrinchus) Fishery Management in the St. Lawrence Estuary, Québec, Canada. In : Williot P. et al. (Ed.), Biology and Conservation of the European Sturgeon Acipenser sturio. Actes de conférence.

Vladykov V. D. 1940 - Nourriture du Marsouin blanc sur les bancs de Manicouagan. Annales de L'ACFAS $6: 120-121$.

Vladykov V. D. 1943 - Studies on aquatic mammals II. A modification of the pectoral fins in the Beluga from the St. Lawrence River, Contribution for the Ministry of Fisheries of the Province of Québec. Le Naturaliste Canadien $70: 23-40$.

Vladykov V. D. 1944 - Studies of aquatic mammals III. Hunting, biology and economic value of the white whale or beluga (Delphinapterus Leucas) of the St. Lawrence River and Gulf. Contribution for the Ministry of Fisheries of the Province of Québec, [En ligne] URL: http://www.dfo-mpo.gc.ca/ Library/111449.pdf. Consulté en mars 2016.

Watson R. A. \& Pauly D. 2013 - The changing face of global fisheries - The 1950s vs. the 2000 s. Marine Policy $42: 1-4$.

Wiber M. G. 2000 - Fishing Rights as an Example of the Economic Rhetoric of Privatization: Calling for an Implicated Economics. Canadian Review of Sociology 37 (3) : 267-288.

\section{NOTES}

1. Il s'agit du mammifère cétacé (Delphinapterus leucas) et non pas d'une des trois variétés d'esturgeon, un poisson, qui porte le même nom (Huso huso).

2. Les Inuit ont le droit de pêcher et de consommer le béluga.

3. L'UICN et Greenpeace ont placé ces espèces sur leur liste rouge. En Europe, elles sont inscrites dans le CITES Appendix II (depuis 1979 pour l'esturgeon et 2007 pour l'anguille) et gérées par l'UE depuis 2009. Aux États-Unis, les anguilles n'ont pas de statut spécial, mais le United States Fish and Wildlife Service et le National Marine Fisheries Service démarchent pour les inscrire sur leur liste d'espèces en danger, alors que les pêches et la consommation d'esturgeons noir sont interdites.

4. En 2010-2011, les pêcheurs ont capturé 9,2\% des anguilles en migration. En 2013, cela a représenté 14 tonnes métriques. En comparaison, les prises atteignaient 1000 tonnes métriques au début du $\mathrm{xx}^{\mathrm{e}}$ siècle et ont oscillé entre 400 et $600 \mathrm{TM}$ annuellement jusque dans les années 1980.

5. Ils étaient plus de 1000 jusqu'à la fin des années 1980 .

6. Les propriétaires des permis restants paient les frais afin de préserver leurs droits de pêche.

7. Le ministère de l'Agriculture, des Pêcheries et de l'Alimentation compte un programme pour le développement de produits certifiés, créé à la suite de la Loi sur les appellations réservées et les termes valorisants (2006). Le MAPAQ doit chercher des produits et des groupes de producteurs 
qui seraient susceptibles de pouvoir s'insérer dans ces procédures et fait donc du démarchage à cet effet.

8. Cela fait suite notamment à l'interdiction de vente de civelles provenant d'Europe sur les marchés internationaux afin d'approvisionner les fermes d'élevage chinoises.

\section{RÉSUMÉS}

Cet article dresse un portrait ethnographique de l'exploitation, de la mise en marché et de la conservation de trois espèces emblématiques de l'estuaire du Saint-Laurent : le béluga, l'anguille et l'esturgeon noir. L'approche de la political ecology mobilisée permet dans un premier temps de retracer l'histoire de la pêche au béluga ayant permis l'avènement des pêches d'anguilles et d'esturgeons. Deuxièmement, le processus de dégradation environnementale qui a mené à des politiques de gestion ayant marginalisé les pêcheurs est examiné. La manière dont ces pêches sont désormais liées à un phénomène de patrimonialisation de la nature et à de nouveaux mécanismes de marchandisation est enfin explorée. Cette analyse permet d'interroger les conséquences environnementales du développement capitaliste et des politiques de gestion de la nature pour les pêcheurs et leur environnement et d'éclairer le processus de précarisation de ces pêches.

This paper presents an ethnographic examination of beluga whale, eel and black sturgeon fisheries, focusing more particularly on their exploitation, commodification and conservation. A political ecology approach first allows for an historical account of the beluga whale fishery, revealing how it contributed to developing the eel and black sturgeon fisheries. Second, the paper examines the environmental degradation process that led to management policies, which are in turn marginalizing the fishermen. Third, it explores how eel and sturgeon fisheries are now tied both to a natural heritagization process and new mechanisms of commodification. This analysis questions the environmental consequences of capitalist development and natural resource management policies for the fishermen and their environment, shedding light on the pressurization process these fisheries are facing.

\section{INDEX}

Mots-clés : pêche, anguille, esturgeon, béluga, political ecology, Québec, conservation

Keywords : fishery, eel, black sturgeon, beluga whale, political ecology, Quebec, conservation

\section{AUTEUR}

\section{SABRINA DOYON}

Professeur titulaire - Département d'anthropologie, Université Laval, Québec, Canada 